\title{
Cogstate Assessment Battery
}

National Cancer Institute

\section{Source}

National Cancer Institute. Cogstate Assessment Battery. NCI Thesaurus. Code C160969.

Five measures to assess for visual learning, executive function, visual attention,

processing speed, and working memory. 